\title{
Effect of Water T2 Shortening in the Quantification of in-vitro Proton MR Spectroscopy
}

\author{
Juan Antonio Hernández-Tamames, Virginia Mato Abad, Roberto García-Álvarez, \\ Javier González-Zabaleta, Javier Pereira Loureiro, Juan Álvarez-Linera
}

From the Laboratorio de Análisis de Imagen Médica y Biometría (LAIMBIO), Universidad Rey Juan Carlos, Madrid, Spain (JAH-T, VMA, JG-Z); Clinical Science Development Group, GE Healthcare, Buc, France, Laboratorio de Imagen Médica y Diagnóstico Radiológico (IMEDIR), Universidad de A Coruña, A Coruña, Spain (JPL); and Hospital Ruber Internacional, Madrid, Spain (JA-L).

\begin{abstract}
Purpose.

This work studies the relationship between in-vitro Proton Magnetic Resonance Spectroscopy metabolite quantification and water T2 decay.

Materials and methods.

An in-vitro correspondence is established between the iron accumulation and the shortening of water T2 relaxation times using seven spherical phantoms, 6 of them were doped with an increasing concentration of iron metal nanoparticles solution. This is later proposed as a source of error during the LCModel metabolite quantification of either absolute concentrations or ratios.

Results

The Pearson's correlation coefficient between water T2 values against absolute metabolite concentrations was on average $[\mathrm{r}]=0.97$ and on average $[\mathrm{r}]=0.85$ for metabolite ratios.

Conclusion

These results suggest that the shortening of T2 values should be taken into account when performing metabolite quantification. Also, the need of demonstrated similar results in in-vivo studies, since the presence of iron deposits or other factors affecting the water T2 decay measurements could explain part of the inter-subject variability in the metabolite concentration and ratio quantification.
\end{abstract}

\section{Keywords:}

Water T2 relaxation time, magnetic resonance spectroscopy, iron, metabolite quantification.

\section{Introduction}

Iron is known to accumulate in the brain as a function of age.[1, 2] However, this process is quite specific and involves the accumulation of iron-containing nuclei, which is one of the components of the basal ganglia. Concentrations of non-haemoglobin iron (mostly ferritin) increase in the putamen, motor cortex, prefrontal cortex, sensory cortex and thalamus during the first 30 to 35 years of life, and variable changes are observed in older individuals.[3] Different studies have shown that levels of H-ferritin in older individuals (67-88 years of age) were higher than in younger controls (27-66 years) in the frontal cortex, caudate nucleus, putamen, substantia nigra and globus pallidus. In the case of L-ferritin, this increase is observed only in the substantia nigra and globus pallidus. [4, 5]

Iron is involved in the mechanisms that underlie many neurodegenerative diseases.[6-12] Conditions such as neuroferritinopathy and Friedreich ataxia are associated with mutations in genes that encode proteins that are involved in iron metabolism, and as the brain ages, iron accumulates in regions that are affected by Alzheimer's and Parkinson's disease. High concentrations of reactive iron can increase 
oxidative-induced neuronal vulnerability, and iron accumulation may increase the toxicity of environmental or endogenous toxins.[2]

The iron effect on the magnetic resonance image (MRI) acquisition can be observed as a local hypointensity.[2, 13, 14] The T2 effect of ferritin is much stronger than the T1 effect.[15] Prominently decreased signal intensity in the globus pallidum, reticular substantia nigra, red nucleus and dentate nucleus was routinely noted in 150 consecutive individuals on T2-weighted images (SE 2000/100) at field strength of 1.5 T.[16] This MR finding correlated closely with the decreasing estimated T2 relaxation times and the location of preferential accumulation of ferric iron using the Perls staining method on normal postmortem brains. The decreased signal intensity on T2-weighted images thus provides an invivo map of the normal distribution of brain iron. Knowledge of the distribution of brain iron should assist in elucidating normal anatomic structures and in understanding neurodegenerative, demyelinating and cerebrovascular disorders.[7, 17]

In proton magnetic resonance spectroscopy $\left({ }^{1} \mathrm{H}-\mathrm{MRS}\right)$, the main interest is to maximize the signal-tonoise ratio of the spectrum peaks, avoiding signal loss due to T1 relaxation and T2 decay.[18] As mentioned before, the presence of iron deposits causes $\mathrm{T} 2$ shortening signal in $\mathrm{T} 2$-weighted images. However, no studies were found in the literature about this effect in the acquisition of the spectrum. This work studies the relationship between in-vitro ${ }^{1} \mathrm{H}-\mathrm{MRS}$ metabolite quantification and water $\mathrm{T} 2$ decay ratios.

\section{Materials and Methods}

In order to assess the in-vitro relationship between the water T2 relaxation times and the spectroscopic data, seven spherical phantoms (phantom1 to phantom7), each $40 \mathrm{~mm}$ in diameter, were filled with identical homogeneous aqueous solutions that mimic the metabolic properties of a healthy human brain. Magnetic nanoparticles with a $\mathrm{Fe}_{3} \mathrm{O}_{4}$ core, coated with dextran and $100 \mathrm{~nm}$ in diameter (Endorem, Guerbert, France), were used to dope phantoms with iron. Increasing concentrations of magnetic nanoparticles were added to an aqueous solution mimicking the metabolic properties of a healthy human brain. Final concentrations of magnetic nanoparticles in phantoms 1 to 7 were: $0 ; 0.5 ; 1.5 ; 2.5 ; 3.5 ; 5$ and $6 \mathrm{mgFe} / \mathrm{ml}$, respectively.

All MR images and spectroscopic data were acquired with a clinical GE Signa HDx 3.0T scanner using a single-channel quadrature head coil. The spectroscopy protocol consisted of a Point Resolved Spin Echo (PRESS) acquisition with TR $=1500 \mathrm{~ms}, \mathrm{TE}=35 \mathrm{~ms}$ and 128 signal averages per data frame with an eight-phase cycling scheme (2,048 data points per spectroscopy frame with a spectral band width of $2,500 \mathrm{~Hz}$ ) resulting in an acquisition time of approximately 3.5 minutes. Suppression of unwanted water signal was accomplished by three chemical shift-selective (CHESS) radio frequency pulses. A single voxel (SV) with a nominal size of $12 \times 12 \times 12 \mathrm{~mm}$ was prescribed at the centre of the phantom. Phantoms were scanned one by one keeping the receiver gain constant from one phantom acquisition to the next. All spectroscopy data were subsequently processed off-line.

Metabolite quantification was performed with the LCModel software.[19] This software fits experimental data with a so-called 'basis set' of spectra. The basis set is acquired from solutions acting as concentration references for the in-vivo acquisitions. This basis set has to be compatible with your data in terms of acquisition parameters such as field strength, localization sequence or TE. The LCModel software has a large collection of basis set for the most common acquisition protocols, like the one used for this work. The concentrations of the pertinent metabolites are determined in LCModel by scaling the relative areas and chemical shifts across the two sets of spectra. Fitting of the spectral peaks is thus achieved with a priori knowledge of their actual characteristics. The main brain metabolites choline (Cho), creatine (Cr), N-Acetyl aspartate (NAA), and myo-inositol (mI) were recorded. As metabolite concentrations are often presented as ratios, the ratios relative to $\mathrm{Cr}(\mathrm{NAA} / \mathrm{Cr}, \mathrm{Cho} / \mathrm{Cr}$ and $\mathrm{mI} / \mathrm{Cr}$ ) were also studied, whose level is explicitly assumed stable in normal as well as in many pathologic conditions.

The water T2 imaging protocol consisted of a contiguous coronal T2-weighted Spin Echo (SE) pulse sequence using 3-mm slice thickness, with FOV $=22 \times 22 \mathrm{~cm}$, matrix size $=256 \times 256$, TR $=1500 \mathrm{~ms}$ and 11 echo times (TE $=20,35,40,60,70,80,105,140,144,216$ and $288 \mathrm{~ms}$ ). Signal intensity (SI) was recorded from the same spatial localization prescribed during the SV acquisition. T2 maps were then computed after mono-exponential fitting of the SI as a function of TE using an in-house developed software.[20] 


\section{Results}

Table 1 shows the water T2 relaxation times for each phantom together with their corresponding concentrations of iron nanoparticles and Figure 1 plots the water T2 relaxation times for each phantom. The T2 relaxation times are clearly seen to decrease as the concentration of iron increases. Line regression (dotted line) in Figure 1 confirms a strong correlation between water T2 and iron concentrations (or phantom number) with a Pearson's correlation coefficient $[\mathrm{r}]=-0.93$.

Table 1. Iron Nanoparticle Concentrations and Water T2 Relaxation Times in each Phantom

\begin{tabular}{lcc}
\hline & Conc. $(\mathrm{mgFe} / \mathrm{ml})$ & $\mathrm{T} 2(\mathrm{~ms})$ \\
\hline & 0 & \\
phantom1 & 0.5 & 153 \\
phantom2 & 1.5 & 125 \\
phantom3 & 2.5 & 88 \\
phantom4 & 3.5 & 65 \\
phantom5 & 5 & 53 \\
phantom6 & 6 & 41 \\
phantom7 & & 37 \\
\end{tabular}

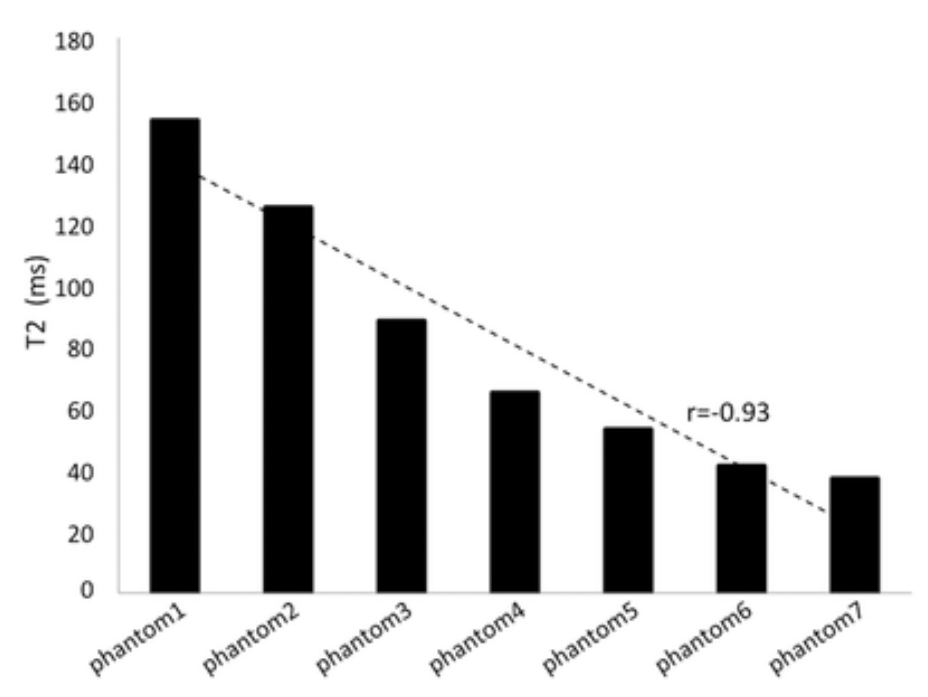

Fig 1. Water T2 relaxation times of each phantom.

Figure 2 demonstrates how reconstructed spectrum (bottom row) is affected by changes in the iron concentration. As the concentration increases, the overall amplitude of the corresponding spectra decreases. This is also accompanied by a decrease in the signal intensity of the water T2 maps (top row). 


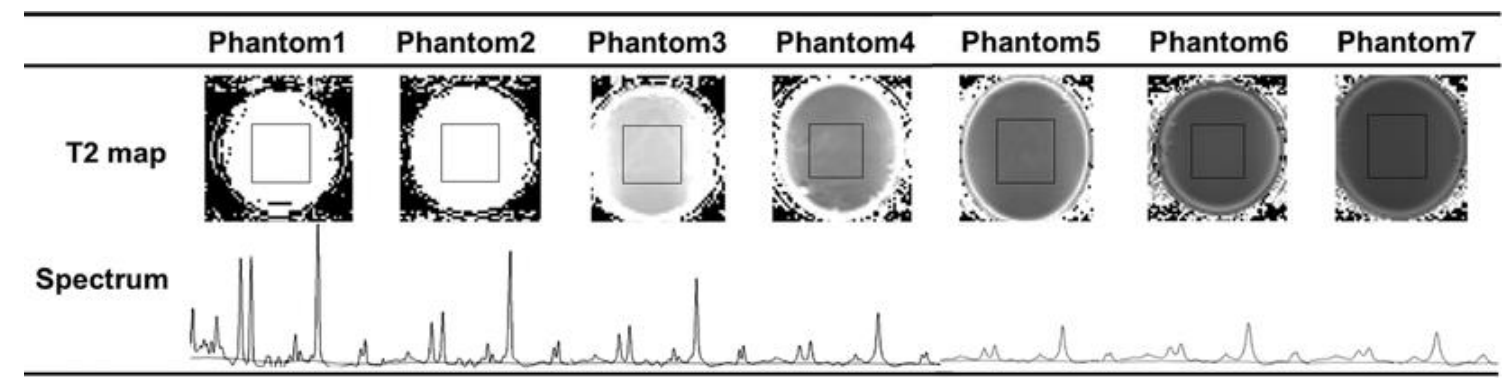

Figure 2. Water T2 maps (top row) and corresponding short echo time (TE $=35 \mathrm{~ms}$ ) spectra (bottom row). All spectra shared the same amplitude scale. Single-voxel spectroscopy excitation borders (solid line) of nominal size $12 \times 12 \times 12 \mathrm{~mm}$ are shown overlaid onto T2 maps.

In Figure 3A, a plot of absolute metabolite concentrations against decreasing water T2 values along the different phantoms is shown. Similarly, Figure 3B displays a plot of metabolite ratios versus decreasing water $\mathrm{T} 2$.
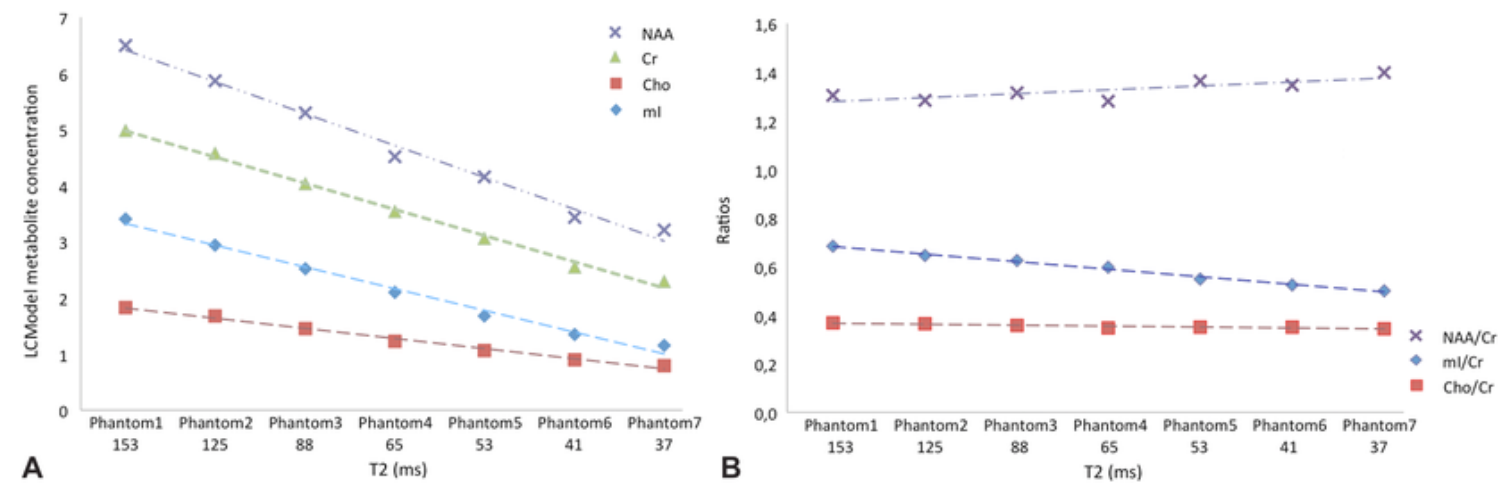

Figure 3. Water T2 values for each phantom versus LCModel absolute metabolite concentrations (A) and ratios (B).

The Pearson's correlation coefficient between the water T2 relaxation times and both the metabolite concentrations and ratios is shown in Table 2. The Pearson's correlation coefficient was on average $[\mathrm{r}]=$ 0.97 and $[\mathrm{r}]=0.85$ for metabolite concentrations and ratios, respectively.

Table 2. The Pearson's Correlation Coefficient [r] between Water T2 Relaxation Times and both the Metabolite Concentrations and Ratios

\begin{tabular}{lc}
\hline Metabolite & {$[\mathrm{r}]$} \\
\hline & \\
$\mathrm{NAA}$ & 0.98 \\
$\mathrm{Cr}$ & 0.97 \\
$\mathrm{mI}$ & 0.98 \\
$\mathrm{Cho}$ & 0.98 \\
$\mathrm{NAA} / \mathrm{Cr}$ & -0.66 \\
$\mathrm{mI} / \mathrm{Cr}$ & 0.96 \\
$\mathrm{Cho} / \mathrm{Cr}$ & 0.93 \\
& \\
\hline
\end{tabular}




\section{Discussion}

The presence of iron nanoparticles causes a signal drop in the computed water T2 maps (Fig 2, top row), which is also accompanied by noticeable changes in the reconstructed LCModel spectra (Fig 2, bottom row). The latter causes underestimation in the absolute metabolite quantification. This effect is also demonstrated in the observed correlation between the water T2 relaxation times and both the metabolite concentrations and ratios (Fig 3).

Magnetic resonance spectroscopy is a valuable diagnostic tool, which, in combination with MRI, enhances tissue characterization of intracranial lesions. Current clinical practice includes the use of metabolite ratios to aid tumor diagnosis and staging. Also, ${ }^{1} \mathrm{H}-\mathrm{MRS}$ is used in clinical practice to identifying evolutionary patterns of regional metabolite abnormalities in neurodegenerative diseases such as Alzheimer's disease and neurological disorders such as Parkinson disease, both of them affected by iron accumulation in the brain. As indicated in Figure 3, the presence of iron deposits could be one of the reasons affecting the accuracy of current spectroscopy diagnosis.

These results suggest that the shortening of T2 values should be taken into account when performing metabolite measurements. Similar results are still to be demonstrated in-vivo, since the presence of iron deposits or other factors affecting the water T2 decay measurements, such as microcalcifications and microbleeds, could explain part of the inter-subject variability in the metabolite concentration and ratio quantification.

These findings show that the T2 values should be considered in clinical practice when performing ${ }^{1} \mathrm{H}$ MRS in pathologies affected by iron accumulation. Not doing so could lead to an underestimation of the metabolite quantification due to the shortening of T2 values, mainly in those metabolites with short T2 water relaxation times only visible with short TE ${ }^{1} \mathrm{H}-\mathrm{MRS}$, such as $\mathrm{mI}$. As shown in Figure 3B, ratios are much less affected to T2 variations, so relative quantification should be routinely used in clinical applications affected by iron accumulation, since correlated effects which influence all metabolite signals to the same degree are potentially corrected by forming ratios. When absolute quantification is required, the imaging protocol should included a iron-sensitive T2-weighted imaging, like SE pulse sequence with multiples echo's or Gradient Echo for T2* relaxation times. The LCModel's absolute concentration for each peak in the spectrum should be proportionally weighted by the T2 values within the spectroscopic voxel. A reference spectrum is usually acquired from the contralateral region of the patient's brain in focal pathologic conditions, such as tumors, to calculate normalized ratios using the metabolic values from the contralateral normal side. This approach could be also applied for the iron-normalization of metabolite concentrations by using brain regions with no preferential iron accumulation as reference for normalization.

The effect of water T2 shortening in ${ }^{1} \mathrm{H}$-MRS quantification could become more important when performing group comparisons in clinical studies involving ${ }^{1} \mathrm{H}$-MRS in elder patients or in pathologies with evident signs of iron accumulation, microcalcifications or microbleeds, since each subject may be affected by the $\mathrm{T} 2$ shortening in different degrees. In this case, it should be necessary to introduce the measurement of water $\mathrm{T} 2$ relaxation times as a new confounding variable in the statistical analysis.

\section{Acknowledgements}

The authors would like to thank Eva Alfayate (Fundación CIEN-Fundación Reina Sofía) for data acquisition.

\section{References}

1. Harder SL, Hopp KM, Ward H, et al. Mineralization of the deep gray matter with age: A retrospective review with susceptibility-weighted MR imaging. Am J Neuroradiol 2008;29:176-83.

2. Zecca L, Youdim MBH, Riederer P, et al. Iron, brain ageing and neurodegenerative disorders. Nat Rev Neurosci 2009;5:863-73.

3. Hallgren B, Sourander P. The effect of aging on the non-haemin iron in the human brain. J Neurochem 1958;3:4151.

4. Connor JR, Snyder BS, Arosi P, et al. A quantitative analysis of isoferritins in select regions of aged, parkinsonian and Alzheimer Disease brains. J Neurochem 1995;65:717-24.

5. Zecca L, Gallorini M, Schünemann V, et al. Iron, neuromelanin and ferritin in substantia nigra of normal subjects at different ages. Consequences for iron storage and neurodegenerative disorders. J Neurochem 2001;76:1766-73.

6. Bartzokis G, Sultzerbc D, Mintzc J, et al. In vivo evaluation of brain iron in Alzheimer's disease and normal subjects using MRI. Biol Psychiatry 1994;35:480-7. 
7. Boddaert N, Sang KH, Rotig A, et al. Selective iron chelation in Friedreich ataxia: biologic and clinical implications. Blood 2007;110:401-8.

8. Dexter DT, Wells FR, Lee AJ, et al. Increased nigral iron content and alterations in other metal ions occurring in brain in Parkinson's Disease. J Neurochem 2006;52:1830-6.

9. Drayer BP, Olanow W, Burger P, et al. Parkinson plus syndrome: diagnosis using high field MR imaging of brain iron. Radiology 1986;159:493-8.

10. Jellinger K, Paulus W, Grundke-Iqbal I, et al. Brain iron and ferritin in Parkinson's and Alzheimer's disease. J Neural Transm Park Dis Dement Sect 1990;2:327-40.

11. Simmons JT, Pastakia B, Chase TN, et al. Magnetic resonance imaging in Huntington disease. Am J Neuroradiol 1986;7:25-8

12. Riederer P, Sofic E, Rausch WD, et al. Transition metals, ferritin, glutathione, and ascorbic acid in parkinsonian brains. J Neurochem 1989;52:515-20.

13. Dormont D, Ricciardi KG, Tandé D, et al. Is the subthalamic nucleus hypointense on T2-weighted images? A correlation study using MR imaging and stereotactic atlas data. Am J Neuroradiol 2004;25:1516-23.

14. Yamada K, González RG, Østergaard L, et al. Iron-induced susceptibility effect at the globus pallidus causes underestimation of flow and volume on dynamic susceptibility contrast-enhanced MR perfusion images. Am J Neuroradiol 2002;23:1022-9.

15. Vymazal J, Righini A, Brooks RA, et al. T1 and T2 in the brain of healthy subjects, patients with parkinson disease, and patients with multiple system atrophy: Relation to iron content. Radiology 1999;211:489-95.

16. Drayer B, Burger P, Darwin R, et al. MRI of brain iron. Am J Roentgenol 1986;147:103-10.

17. Schenck JF, Zimmerman EA. High-field magnetic resonance imaging of brain iron: birth of a biomarker? NMR Biomed 2004;17:433-45.

18. McRobbie DW, Moore EA, Graves MJ, Prince MR. MRI from picture to proton. Cambridge University Press, New York; 2006.

19. Provencher SW. Estimation of metabolite concentrations from localized in vivo proton Nmr-spectra. Magn Reson Med 1993;30:672-9.

20. González-Zabaleta J, Malpica N, Álvarez-Linera J, et al. Software platform for brain T2 relaxometry quantification (abstract). MAGMA 2009;22(Issue 1 Suppl):541. 